\title{
The dichotomy between strong and ultra-weak magnetic fields among intermediate-mass stars
}

\author{
François Lignières $^{1,2}$, Pascal Petit ${ }^{1,2}$, Michel Aurière ${ }^{1,2}$, Gregg A. \\ Wade $^{3}$ and Torsten Böhm ${ }^{1,2}$ \\ ${ }^{1}$ CNRS, Institut de Recherche en Astrophysique et Planétologie \\ 14 avenue Edouard Belin, 31400 Toulouse, France \\ email: francois.lignieres@irap.omp.eu \\ ${ }^{2}$ Université de Toulouse, UPS-OMP, IRAP \\ 31400 Toulouse, France \\ ${ }^{3}$ Department of Physics, Royal Military College of Canada \\ PO Box 17000, Station Forces, Kingston, Ontario K7K 7B4, Canada
}

\begin{abstract}
Until recently, the detection of magnetic fields at the surface of intermediate-mass main-sequence stars has been limited to Ap/Bp stars, a class of chemically peculiar stars. This class represents no more than $5-10 \%$ of the stars in this mass range. This small fraction is not explained by the fossil field paradigm that describes the Ap/Bp type magnetism as a remnant of an early phase of the star-life. Also, the limitation of the field measurements to a small and special group of stars is obviously a problem to study the effect of the magnetic fields on the stellar evolution of a typical intermediate-mass star.

Thanks to the improved sensitivity of a new generation of spectropolarimeters, a lower bound to the magnetic fields of $\mathrm{Ap} / \mathrm{Bp}$ stars, a two orders of magnitude desert in the longitudinal magnetic field and a new type of sub-gauss magnetism first discovered on Vega have been identified. These advances provide new clues to understand the origin of intermediate-mass magnetism as well as its influence on stellar evolution. In particular, a scenario has been proposed whereby the magnetic dichotomy between $\mathrm{Ap} / \mathrm{Bp}$ and Vega-like magnetism originate from the bifurcation between stable and unstable large scale magnetic configurations in differentially rotating stars. In this paper, we review these recent observational findings and discuss this scenario.
\end{abstract}

Keywords. Stars : magnetic fields, instabilities

\section{Introduction}

The origin of the stellar magnetic fields and their effects on the star structure and evolution are the two basics questions of stellar magnetism. In all stars that possess a convective envelope, a magnetic field is believed to be generated by dynamo mechanism. It is not always possible to detect it, but its presence at the surface of these solartype stars makes little doubt. For these stars, we also know that a magnetized wind exerts a breaking torque that strongly affects their angular momentum evolution. This illustrates that although limited our level of understanding of the magnetic fields of solar-type stars enables to answer at least some simple questions about their origin and their impact. This is not the case for hotter stars with radiative envelope where the origin of the observed fields as well as their impact on the star evolution remain largely mysterious. The properties of the observed fields are nevertheless well established. They are large scale mostly dipolar fields with dipole strength ranging from $300 \mathrm{G}$ to $30 \mathrm{kG}$ and are remarkably stable over time (Donati \& Landstreet, 2009). These fields are usually 
called fossil fields as a reference to the hypothesis that describes them as remnant of an early phase of the star-life, either from the collapse of a magnetized cloud or during the convective protostellar phase. But they only are detected in a small $5-10 \%$ fraction of the intermediate-mass and massive stars and this fact did not receive a convincing explanation yet. From the point of view of stellar evolution, such a lack of understanding of the field generation processes combined with the absence of direct measurements for the vast majority (90-95\%) of stars are a major obstacle to model the effect of a magnetic field on the structure and evolution of typical intermediate-mass and massive stars.

In this paper, we review recent observational results that shed a new light on the $5-10 \%$ problem and suggested new scenarios for the origin of hot star magnetism. These results have been obtained thanks to the high sensitivity of a new generation of high-resolution échelle spectropolarimeters (MuSiCoS, Narval, ESPaDOnS, HARPSpol). In particular, their large wavelength coverage allows to sum up the magnetically polarized signal of many spectral lines and thus to detect weak fields. The number of lines available being an important factor, it is comparatively easier to detect weak fields in cooler stars. For example, Aurière et al. (2009) showed that a sub-gauss longitudinal field can be detected on the bright late-type star Pollux averaging only a few high S/N Stokes V of Narval or ESPaDOnS. For the same reason, weak fields are easier to detect in intermediate-mass stars than in more massive stars.

Spectropolarimetric surveys of Ap stars as well as of A-type non-Ap stars have first ruled out the possibility that the $5-10 \%$ fraction simply reflects the detection limit of magnetic measurements. Indeed, the magnetic fields of $\mathrm{Ap} / \mathrm{Bp}$ stars were found to be higher than a lower limit of the order of $100 \mathrm{G}$ for the longitudinal field while no field were detected in A-type non-Ap stars. Then two detections of sub-gauss fields on the bright A stars Vega and Sirius have been obtained. Thus, instead of a single class of magnetic stars, intermediate-mass star magnetism is now characterized by two type of magnetisms, Ap/Bp and Vega-like, separated by a two orders of magnitude magnetic desert between $1 \mathrm{G}-100 \mathrm{G}$. In section 2, we present the evidences for the lower bound to the Ap/Bp magnetic fields and the magnetic desert, then the discovery of a new type of sub-gauss magnetism is described in section 3. The implications for the origin of upper-main-sequence magnetism are discussed in section 4 .

\section{The lower bound of Ap/Bp magnetic fields and the magnetic desert}

From the first Zeeman effect measurement (Babcock 1947) to the early 90s, all magnetic field detections in the upper-main-sequence have been achieved among the Ap/Bp stars, a group of late B, A and early F stars showing strong chemical peculiarities. The vector modulus measured from the line broadening in the intensity spectrum ranged from 2 and $30 \mathrm{kG}$ while measurements of the circular polarization of spectral lines allowed to find weaker fields, down to $\sim 300 \mathrm{G}$ dipolar fields.

At that time, the detection limit was too high to study Ap/Bp stars with weak magnetic fields or to put strong constraints on the upper bound of the magnetic fields in A-type non Ap/Bp stars (Landstreet 1982). Thus, although this was suspected, it was not possible to confirm that all $\mathrm{Ap} / \mathrm{Bp}$ stars were magnetic. Moreover a low-field continuation of the $\mathrm{Ap} / \mathrm{Bp}$ magnetism among non-Ap/Bp stars could not be ruled out.

This last point is neither excluded from our understanding of the link between the chemical anomalies and magnetic fields in Ap/Bp stars. Michaud et al. $(1970,1976)$ indeed proposed that the magnetic fields of $\mathrm{Ap} / \mathrm{Bp}$ stars are strong enough to avoid macroscopic mixing in the envelope either through differential rotation, thermal 
convection or stellar winds. He showed that in the quiescent outer layers microscopic diffusion processes produce strong anomalies in the chemical abundances compatible with those observed at the surfaces of Ap/Bp stars. This model also explains why chemical anomalies are either absent (in normal stars) or reduced (in the slowly rotating Am stars) when no fields are detected. Following this picture, we can conclude that all Ap/Bp stars should possess a magnetic field higher than the minimum field strength required to suppress macroscopic mixing and that, if a magnetic field is present at the surface of A-type non-Ap/Bp stars, its strength should be smaller than this minimum field.

From the mid-90s, deep surveys have been conducted with the spectropolarimeters MuSiCoS and Narval at Telescope Bernard Lyot to explore the weakly magnetic Ap/Bp stars and non Ap/Bp stars. Aurière et al. (2007) selected a sample of $28 \mathrm{Ap} / \mathrm{Bp}$ stars for which previous attempts had led to no detection or to unreliable ones. Thanks to the improved sensitivity of these instruments, a magnetic field was found in all the stars of the sample confirming that all Ap/Bp stars are magnetic. Moreover, this survey proved the existence of a lower bound to the magnetic field of Ap/Bp stars. The maximum absolute value reached by the longitudinal field as the star rotates $B_{L}^{\max }$ is higher than $\sim 100 \mathrm{G}$, while the dipolar fields obtained by fitting an oblique rotator model - a magnetic dipole inclined with respect to the rotation axis - is higher than $B_{\min }=300 \mathrm{G}$ in all but two stars for which the best model is slightly lower but still compatible with the $300 \mathrm{G}$ value within the error bars.

This result is fully compatible with the idea that a minimum field strength is needed to produce the Ap/Bp chemical anomalies. But one must keep in mind that the observed lower bound of $\mathrm{Ap} / \mathrm{Bp}$ magnetic fields could be either higher or equal to this minimum field. If it is equal, then one would expect to find a low field extension of the $\mathrm{Ap} / \mathrm{Bp}$ magnetism among the other intermediate-mass stars. Indeed, it is difficult to imagine that a mechanism only generates magnetic fields in a sub-group of stars and that, in addition, the smallest field generated this way is exactly the minimum field required for Ap/Bp chemical anomalies.

The simplest extension one could think of is a population of dipolar-like fields that continuously extends the distribution of Ap/Bp dipolar fields below $300 \mathrm{G}$. However, surveys among A and late-B stars conducted with MuSiCoS, Narval and HARPSpol (Shorlin et al. 2002, Aurière et al. 2010, Makaganiuk et al. 2011) as well as deep spectropolarimetric runs dedicated to specific objects (e.g. Wade et al. 2006, Kochukhov et al. 2011), have ruled out the existence of such a population. These non-detections instead revealed a gap in longitudinal field between $100 \mathrm{G}$, the lower bound of Ap/Bp magnetism, and the detection limit of the surveys. With MuSiCoS, Shorlin et al.(2002) reached a detection limit of the order of $50 \mathrm{G}$ for the longitudinal fields, while the Narval survey (Aurière et al. 2010) set a $3 \sigma$ upper limit of the longitudinal field to $10 \mathrm{G}$ for most stars of the sample down to $\sim 1 \mathrm{G}$ in some bright low v sini targets like Sirius. In the following, we shall speak of the magnetic desert in longitudinal field to describe this gap.

Its discovery among intermediate-mass stars has important consequences : The first one is that $\mathrm{Ap} / \mathrm{Bp}$ stars constitute a separated class of stars in what concerns their magnetic properties because no low field continuous extension of this large-scale stable magnetism could be found among intermediate-mass stars. The so-called $10 \%$ problem of why only a small fraction of stars appears to be magnetic is therefore not due to an observational bias but corresponds instead to a true physical dichotomy between Ap magnetism and other intermediate-mass stars. The lower bound of Ap/Bp star magnetic fields must thus be regarded as a characteristic property of $\mathrm{Ap} / \mathrm{Bp}$ magnetism whose 


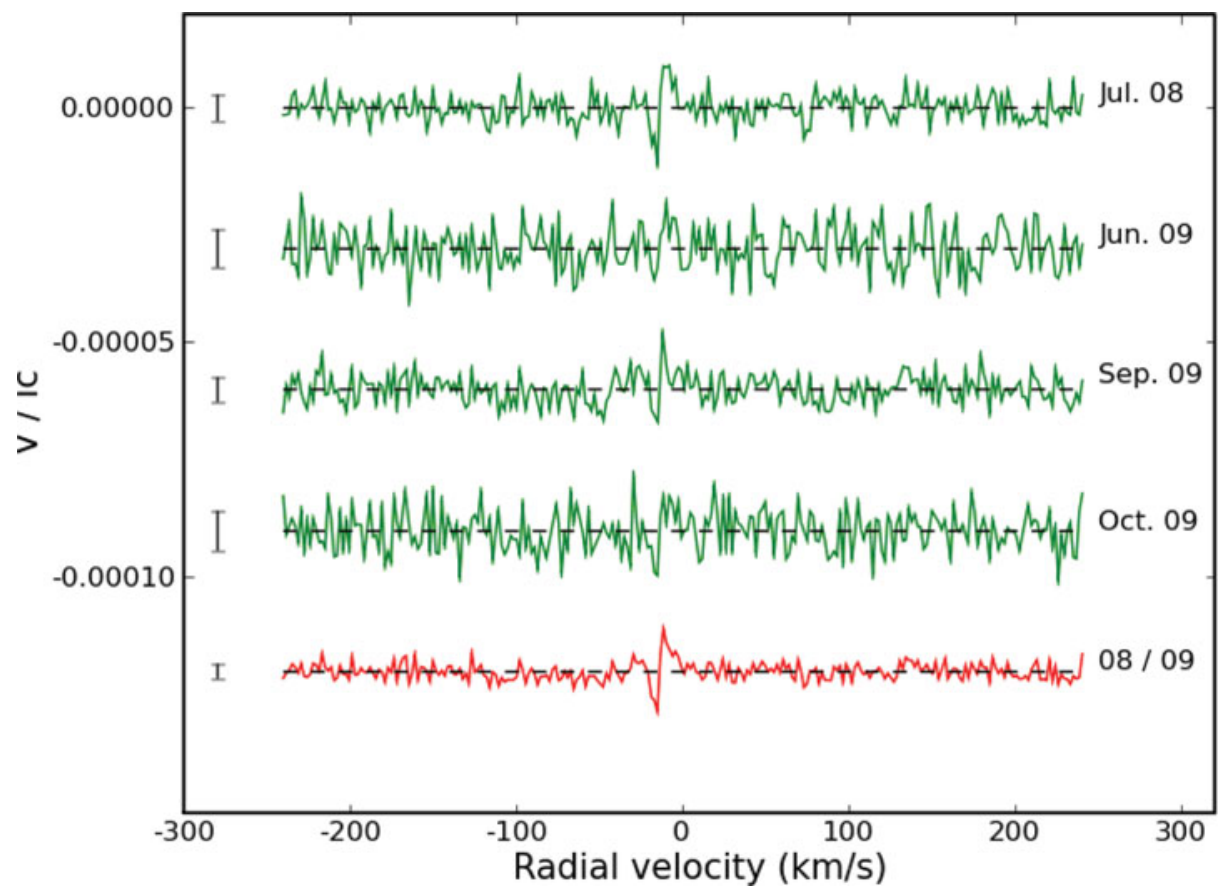

Figure 1. Averaged Stokes V LSD profiles of Vega for 4 different observing runs from July 2008 to October 2009. The red profile is obtained by averaging all 799 spectra taken over this period.

dependency on the star fundamental parameters should provide useful insight into the origin of this magnetism. We shall come back to this point in section 3 .

\section{Ultra-weak magnetic field}

While revealing a magnetic desert among intermediate-mass stars, the spectropolarimetric surveys mentioned in the previous section could only provide an upper bound of the surface magnetic field of A-type non-Ap/Bp stars. Fortunately, a 4-nights Narval run designed to search for pulsations on the A0 star Vega was used as an opportunity to further lower this bound and resulted in the detection of a very small polarimetric signal. The peak Stokes $\mathrm{V}$ amplitude divided by the continuum intensity was $V / I_{c}=10^{-5}$, for a noise level of $\sigma=2 \times 10^{-6}$. The corresponding longitudinal magnetic field is $-0.6 \pm 0.3$ $\mathrm{G}$ (Lignières et al. 2009). This weak signal called for additional measurements and tests to confirm the presence of a magnetic field. As illustrated on Figure 1, additional observing campaigns conducted with Narval and ESPaDOnS over three years all confirmed this first detection (Petit et al. 2010, Alina et al. 2012). The possibility of a spurious detection due to the instrument or the reduction process is systematically tested by computing the combination of sub-exposures, the so-called null profiles, for which the signal is expected to cancel. On the other hand, if the signal is of stellar origin, it should be sensitive to the Landé factor of the spectral lines as well as periodically modulated by the star rotation. Figure 2 shows the Stokes V LSD profiles computed for two lines lists having different mean Landé factors. As expected for a Zeeman signature, the amplitude ratio between the two Stokes V profiles is close to the 1.6 ratio of their mean Landé factor. A periodic modulation at $0.678 \pm 0.036$ day has also been detected by applying a least squares sine fit period search to each radial velocity bin of the Stokes V LSD profile 


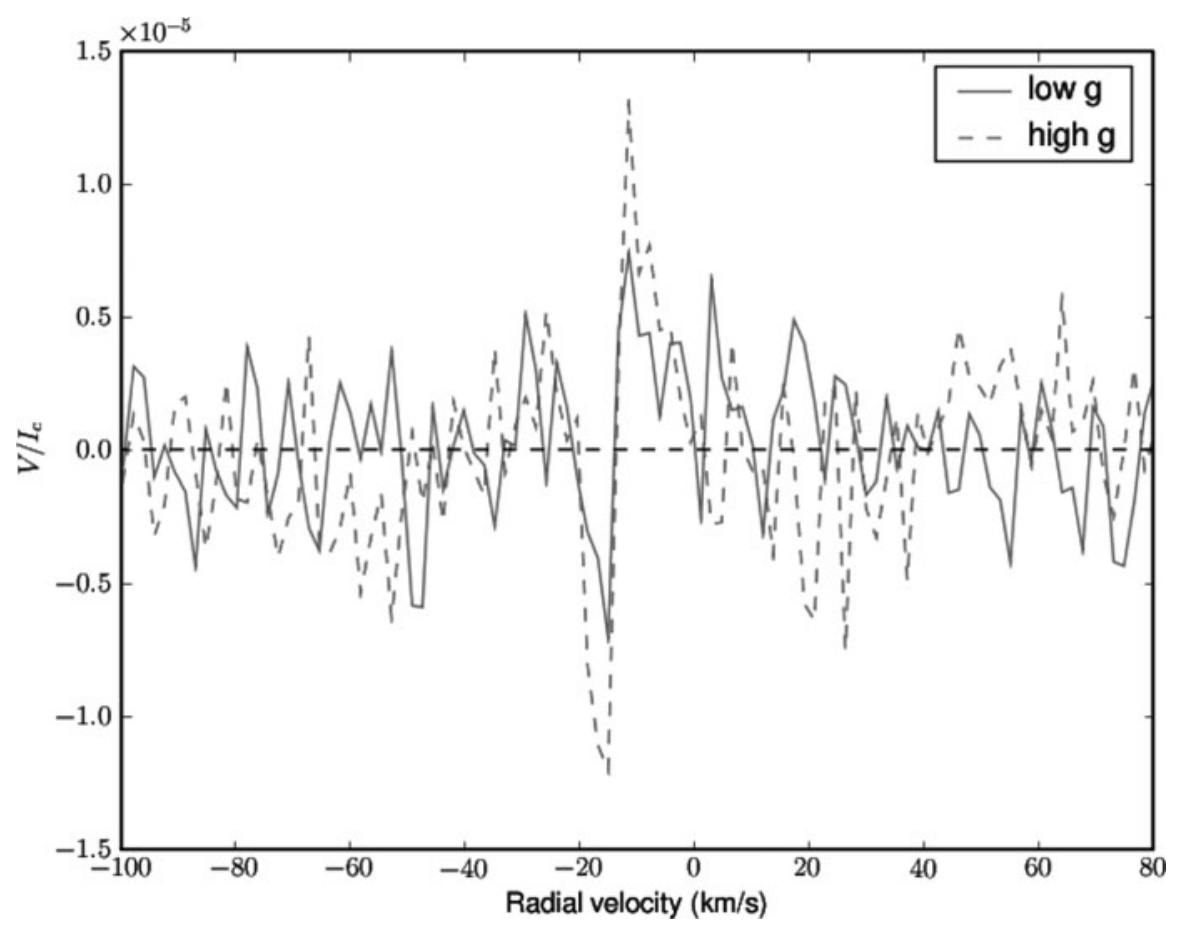

Figure 2. (Colour online) Averaged Stokes V LSD profiles of Vega for two different lines lists. The green and solid profile corresponds to the line list with low Landé factor, the red and dashed profile to high Landé factor. As expected for a Zeeman signal, the amplitude ratio between the two Stokes V profiles is close to the 1.6 ratio between the mean Landé factor of the line lists.

(Alina et al. 2012). This value can be compared with two different determinations of the rotation rate that both make use of the gravity darkening effect, that is the effect of the centrifugal force on the local atmospheric parameters. The line shapes fitting by Takeda et al. (2008) provided 0.7-0.9 day for the rotation period together with a small 7 degrees inclination angle. By contrast the first model fitting of the interferometric observations gave much higher close-to-break-up periods (0.5-0.6 days) with nevertheless similar inclination angles. In a recent study however, Monnier et al. (2012) showed that the error bars of these interferometric determinations were underestimated and a more accurate value now consistent with spectropolarimetric and spectroscopic inferences was obtained thanks to better phase coverages and angular resolutions. Accordingly, the equatorial velocity is $194 \pm 6 \mathrm{~km} \mathrm{~s}^{-1}$, a value only slightly above the average equatorial velocity of normal A0-A1 stars $\mathrm{v}_{\mathrm{eq}}=165 \mathrm{~km} \mathrm{~s}^{-1}$ taken from Royer et al. (2007).

Finding the rotation period is key to reconstruct the surface magnetic field through Zeeman-Doppler Imaging. The magnetic field vector appears to be dominated by its radial component whose surface distribution is structured at small lengthscale and characterized by a slightly off-axis polar spot, as seen on Figure 3. This polar spot can already be inferred from the phase-averaged Stokes V concentration in the weakly Doppler-shifted part of the profile.

If we exclude the debated field detections obtained with the low-resolution FORS1/2 instrument at VLT (e.g. Bagnulo et al. 2012, Kochukhov 2013), Vega is the first detected magnetic A star which is not an Ap/Bp chemically peculiar star. Its magnetic field distinguishes clearly from $\mathrm{Ap} / \mathrm{Bp}$ magnetic fields by the strength of the longitudinal component (two orders of magnetic lower than the minimum longitudinal field of $\mathrm{Ap} / \mathrm{Bp}$ 

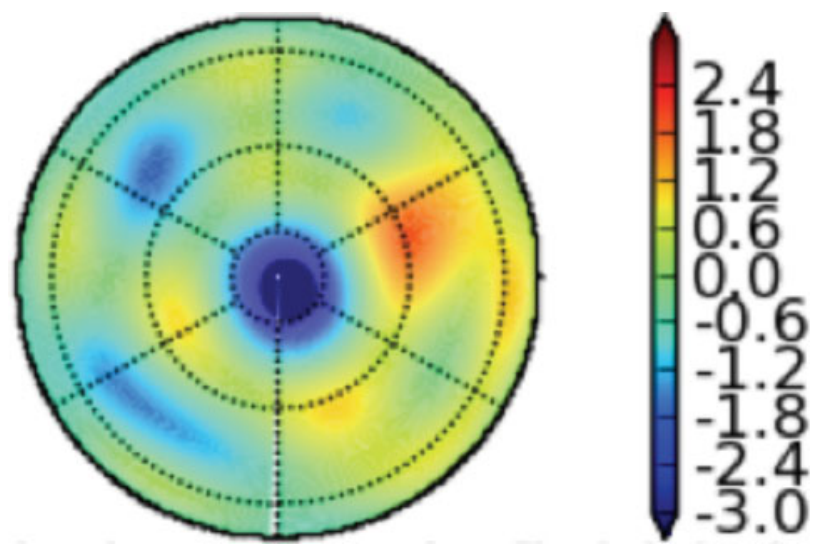

Figure 3. (Colour online) Zeeman-Doppler Imaging of Vega for 2008 July showing the radial component of the magnetic field in polar projection. The color code is expressed in gauss. The spot observed near the pole can be related to the near-zero Doppler velocity feature of the Stokes V profile.

stars taken at its maximum phase) and by the lengthscale of its surface distribution (significantly smaller than for a dipole). It shows that a new - and potentially widespread - class of magnetic stars exists at the low end of the A-type magnetic desert.

The next obvious target is Sirius A, a low v sini bright A1 star with Am-type chemical abundance anomalies. The LSD Stokes V profile obtained by Petit et al. (2011) from the average of 442 high $\mathrm{S} / \mathrm{N}$ ratio spectra clearly shows a polarimetric signal. The $V / I_{c}$ maximum amplitude is similar to Vega but, contrary to Vega, the V profile is asymmetric about the line center. Asymmetric profiles are not standard although they are observed in some cool stars (Petit et al. 2005, Aurière et al. 2009) and locally on the Sun surface where they are interpreted as due to the presence of both magnetic field and velocity field radial gradients in the emitting regions (Lopez-Ariste 2002). A model of stellar atmosphere that produces an asymmetric profile similar to Sirius on a global scale is still lacking. Moreover, the quality of the data has not allowed yet to show a significant sensitivity to the Landé factor or a temporal modulation of the polarimetric signal. Thus, by contrast with Vega, more theoretical and observational works are required to confirm the presence of a magnetic field on Sirius. A spurious detection seems however unlikely as the $\mathrm{V}$ profile found with ESPaDOnS and Narval has been recently recovered using HARPSpol (see Kochukhov 2013). The rotation of Sirius is not known but as the equatorial velocities of Am stars are always smaller than $120 \mathrm{~km} \mathrm{~s}^{-1}$ (Abt 2009) we know that Sirius rotates more slowly than a typical A0-A1 star.

An ongoing Narval survey investigates the occurrence of Vega-like magnetism among tepid stars. The weakness of the expected signal, the necessity to avoid confusion with hypothetical weak solar-type dynamo field in colder stars, the drastic decrease in the number of lines towards hotter stars and the willingness to avoid a bias towards the slowly rotating Am stars contributed to construct a sample of 10 bright stars restricted to the A0-A2 range (including Vega and Sirius). This survey also intends to investigate how Vega-like magnetism depends on rotation and time. A large occurrence of magnetic field detections would have a direct impact on stellar evolution model by providing the first direct constraint on the value of the magnetic field of a typical intermediate-mass star. Meanwhile, the hypothesis of a widespread magnetism is suggested by the analysis of the Kepler photometry of thousands of A stars. Indeed, according to Balona (2011), a low frequency modulation of the light-curve compatible with a rotational modulation has 


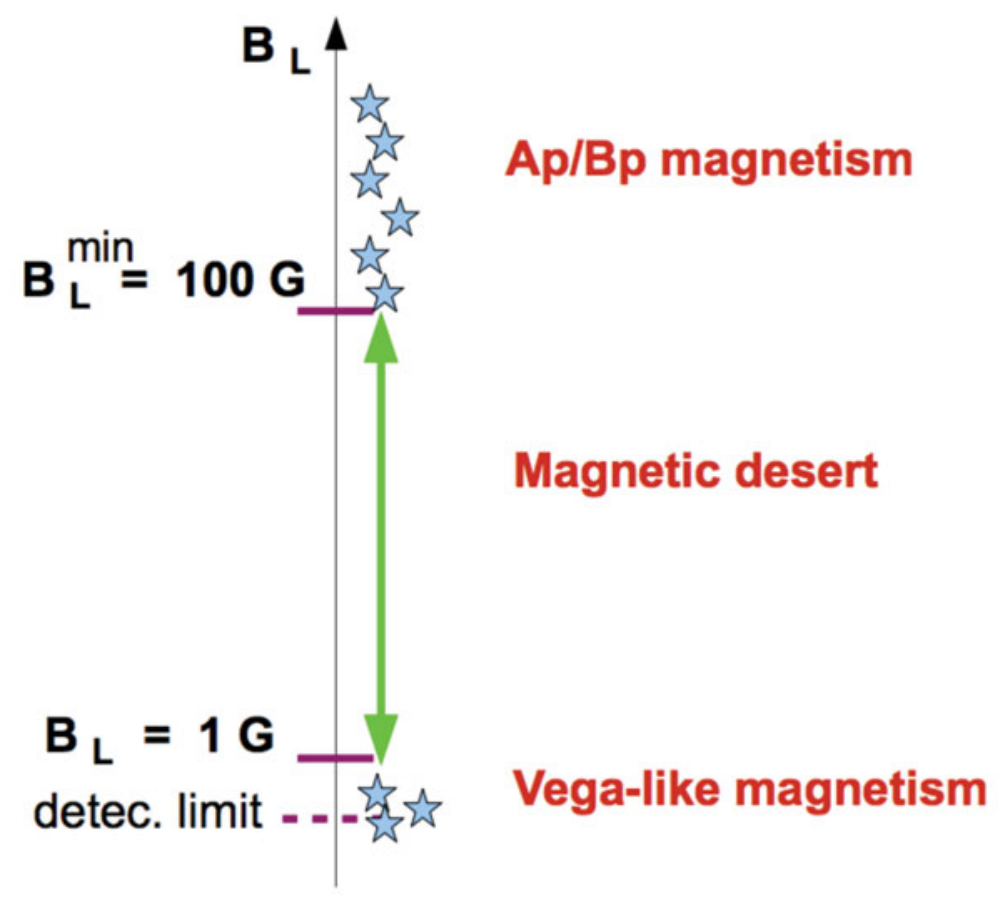

Figure 4. (Colour online) This sketch summarizes the new context of intermediate-mass magnetism set by the recent discoveries of the lower bound of Ap magnetism, the magnetic desert in longitudinal field $B_{L}$ and the Vega-like magnetism.

been found in $70 \%$ of the A-type Kepler stars, as expected in the presence of starspots or other magnetic corotating features.

\section{On the origin of the magnetic dichotomy}

The sketch of Fig. 4 illustrates the dichotomy between the Ap/Bp-like and Vega-like magnetic fields of intermediate-mass stars. One can think of two different ways to explain this dichotomy : the first one is to assume that the two types of magnetic fields have different properties because they have been generated by two different processes. Another possibility is that the observed fields have a common origin but during the evolution their magnetic field distribution split into two distinct families of low and high longitudinal fields.

Braithwaite \& Cantiello (2013) proposed that Vega-like stars are "failed fossil" magnetic stars meaning that their field, produced during star formation, is still decaying thus not truly fossil. Accordingly, the helicity of the initial field configuration must be low enough to evolve towards a sub-gauss field amplitude at the age of Vega. By contrast, the initial helicity of Ap/Bp-like magnetic stars has to be very high to produce in a relatively short time the observed fossil-like Ap/Bp magnetism. Braithwaite \& Cantiello (2013) thus argue that two distinct generation mechanisms must be invoked to explain these very different initial helicities. To account for $\mathrm{Ap} / \mathrm{Bp}$ stars, they follow Ferrario et al. (2009) and Tutukov\& Fedorova (2010) who assume that these stars result from the merging of close binaries, their strong fields being produced by a powerful dynamo during the merging phase. 


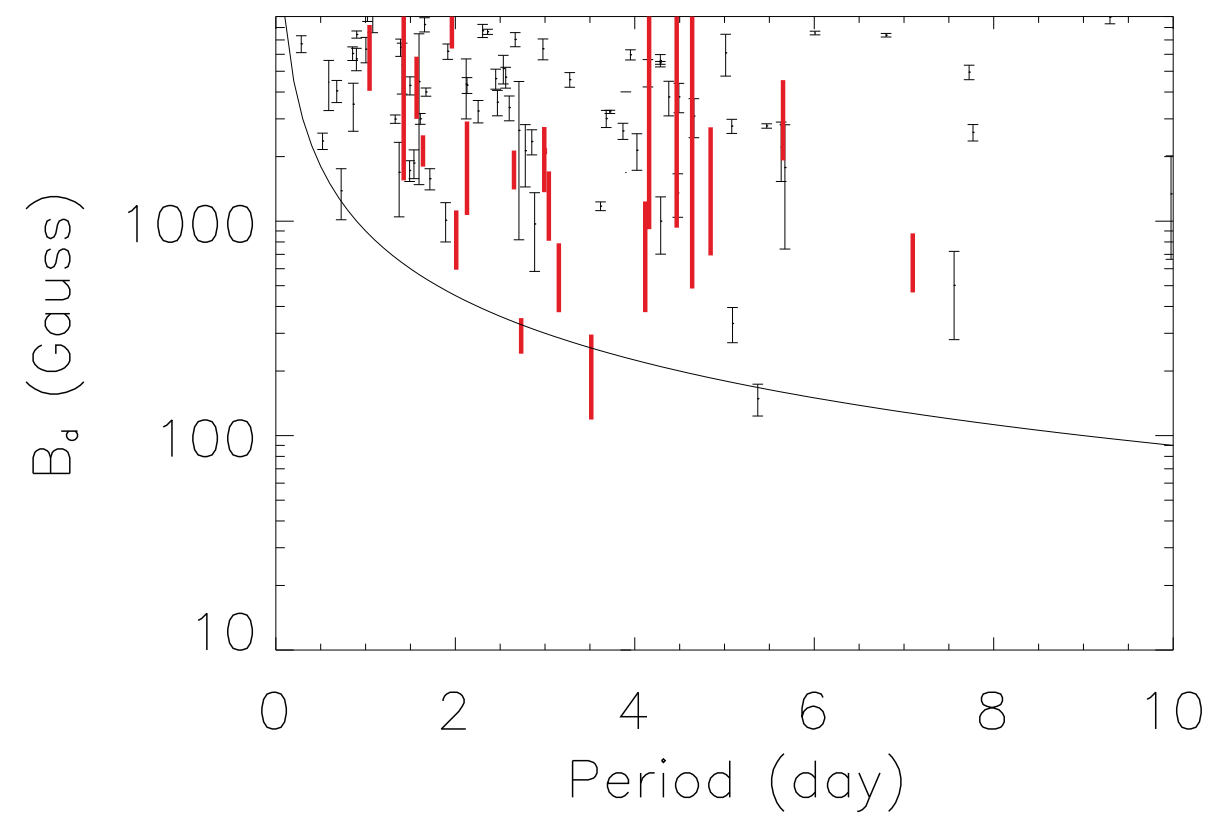

Figure 5. (Colour online) Ap/Bp dipolar field strengths as a function of the rotation rate. The black vertical line segments are dipole strength (with $1 \sigma$ error bars) derived from the longitudinal field measurements of the Bychkov's catalogue and the inequality : $B_{d}>3.3 B_{L}^{\max }$. The red segments correspond to dipole strength (with $1 \sigma$ error bars) obtained by fitting an oblique rotator model (taken from Aurière et al. 2007). The black continuous line is a $B_{d} \propto \Omega$ curve.

On the other hand, Aurière et al. (2007) proposed a bifurcation scenario by considering the evolution of a differentially rotating star as a function of its rotation rate and its poloidal field strength. Weak initial poloidal fields cannot prevent their winding-up by differential rotation into strong toroidal fields. The increasingly toroidal configuration is expected to become unstable to an instability, like the Tayler instability, transforming the initially large scale field configuration into a new configuration with opposed signed polarities at the length scale of the instability. On the contrary, if the initial magnetic field is strong enough, Maxwell stresses impose uniform rotation and eventually lead to a stable configuration. Thus, starting from a continuous distribution of poloidal field strength ranging from low to high dipole strengths, this mechanism predicts a sharp decrease of the longitudinal field between the stable large-scale high $\mathrm{B}_{L}$ fields and the unstable configurations where $\mathrm{B}_{L}$, the surface averaged line-of-sight component, is strongly reduced due to the cancellation of the opposed polarities. In support of this scenario, the observed $\sim 300 \mathrm{G}$ lower bound of Ap/Bp magnetic fields (see section 2) happens to be close to an order of magnitude estimate of the critical dipolar field separating the stable and unstable configuration, namely $B_{c}=(4 \pi \rho)^{1 / 2} r \Omega$, computed for the parameters of a typical Ap star $\left(\log g=4, T_{\text {eff }}=10^{4} K, P_{\text {rot }}=5\right.$ days $)$.

Another important property of $\mathrm{B}_{c}$ is to be proportional to $\Omega$. Thus if this scenario can explain the dichotomy, the lower bound of $\mathrm{Ap} / \mathrm{Bp}$ magnetic fields should increase with $\Omega$. This can be tested since the spectropolarimetric monitoring of an Ap/Bp star gives access to both the rotation period and the dipole strength. The difficulty is that finding the minimum dipolar fields of $\mathrm{Ap} / \mathrm{Bp}$ stars for various rotation rate intervals requires a significant sample of $\mathrm{Ap} / \mathrm{Bp}$ stars for each rotation range. As a first attempt, Aurière \& Lignières (2013) compiled data from the Bychkov catalogue (Bychkov et al. 2005) 
although in many cases the data are not good enough for an oblique rotator modelling. Nevertheless with the maximum value of the longitudinal field $\mathrm{B}_{L}^{\max }$ available, a lower limit of the dipole strength can be determined from the relation $B_{d}>3.3 B_{L}^{\max }$ derived from the oblique rotator model. These lower limits are displayed on Fig. 5 with errors bars together with $B_{d}(\Omega)$ values taken from Aurière et al. (2007). The results seem compatible with a $\mathrm{B}_{\min } \propto \Omega$ law. This data sample is however too limited to obtain an accurate determinations of $B_{\min }$, specially for rapid $A p / B p$ rotators. This calls for a comprehensive investigation of the $\mathrm{B}_{\mathrm{min}}$ dependency on the rotation rates. More generally, we should expect that this lower bound, and its dependency on the stellar parameters, contains important information about the origin of Ap/Bp magnetism.

The physical grounds of the Aurière et al. scenario can also be investigated through 2D and 3D numerical simulations. The winding-up of a poloidal field by differential rotation is accessible to 2D axisymmetric simulations while the non axisymmetric instabilities of the wounded-up configuration require full 3D simulations (e.g. Arlt \& Rüdiger 2011). Among the open questions is the mechanism that drives differential rotation and whether this mechanism is efficient enough to overcome Maxwell stresses. The seismic analysis of sub-giant stars has shown recently that differential rotation is indeed present in the contracting/expanding radiative interiors of these stars (Deheuvels et al. 2012). This points towards pre-main-sequence contraction as a possible source for the differential rotation forcing required in the Aurière et al. scenario.

\section{Conclusion}

While some years ago the fraction of intermediate-mass stars hosting large-scale stable magnetic fields like Ap/Bp stars was unknown, this class of magnetic stars is now known to be clearly separated from the other stars by a two order of magnitude magnetic desert in longitudinal field down to a new type of sub-gauss magnetism. This paradigm shift opens new perspectives for both observational and theoretical studies. Investigating how the lower bound of Ap/Bp magnetic fields depends on the stellar parameters could help to discriminate between the different hypothesis regarding the origin of this magnetism. For example we have seen that the Aurière et al. scenario predicts that this lower bound is proportional to the rotation rate. Moreover, the nature and the incidence of Vegalike magnetism among non $\mathrm{Ap} / \mathrm{Bp}$ stars need to be elucidated although detecting many sub-gauss fields in A stars will be very demanding in observing time. Another important question is whether the dichotomy of intermediate-mass star magnetism is also present among pre-main-sequence intermediate-mass stars and massive stars. This is already suggested by recent spectropolarimetric surveys that detected fossil-like magnetic fields with an incidence similar to Ap/Bp stars (Alecian et al. 2013, Wade et al. 2013).

Various scenarios have been proposed to account for the magnetic dichotomy. Here we described the bifurcation between stable and unstable magnetic configurations and briefly mentioned the merging scenario for Ap/Bp stars and the failed fossil scenario for Vega-like magnetism (see the contributions by N. Langer and J. Braithwaite in these proceedings for more details). Besides the confrontation with observations, numerical simulations will also help to test these ideas. MHD computations in polytropic stars already enabled to find stable magnetic configurations that might correspond to $\mathrm{Ap} / \mathrm{Bp}$ stars (Braithwaite 2009). But, except for very strong fields, differential rotation should also play an important role in shaping magnetic configurations in radiative interiors and this effect needs to be fully explored numerically. 


\section{Acknowledgements}

The authors thank the ANR project IMAGINE and the PNPS (Programme National de Physique Stellaire) of INSU for their financial support.

\section{References}

Abt, H. A. 2009, AJ, 138, 28

Alecian, E. 2013, arXiv:1310.1725

Alina, D., Petit, P., Lignières, F., et al. 2012, AIP Conference Series, 1429, 82

Arlt, R. \& Rüdiger, G. 2011, MNRAS, 412, 107

Aurière, M. \& Lignières, F. 2013, private communication

Aurière, M., Wade, G. A., Lignières, F., et al. 2010, A\&3A, 523, A40

Aurière, M., Wade, G. A., Konstantinova-Antova, R., et al. 2009, A\&AA, 504, 231

Aurière, M., Konstantinova-Antova, R., Petit, P., et al. 2008, A\& A, 491, 499

Aurière, M., Wade, G. A., Silvester, J., et al. 2007, A\&A, 475, 1053

Babcock, H. W. 1947, ApJ, 105, 105

Bagnulo, S., Landstreet, J. D., Fossati, L., \& Kochukhov, O. 2012, A\&3A, 538, A129

Balona, L. A. 2011, MNRAS, 415, 1691

Braithwaite, J. \& Cantiello, M. 2013, MNRAS, 428, 2789

Braithwaite, J. 2009, MNRAS, 397, 763

Bychkov, V. D., Bychkova, L. V., \& Madej, J. 2005, A\& A, 430, 1143

Donati, J.-F. \& Landstreet, J. D. 2009, ARA\& $A, 47,333$

Deheuvels, S., García, R. A., Chaplin, W. J., et al. 2012, textitApJ, 756, 19

Ferrario, L., Pringle, J. E., Tout, C. A., \& Wickramasinghe, D. T. 2009, MNRAS, 400, L71

Kochukhov, O., Makaganiuk, V., Piskunov, N., et al. 2011, A\&A, 534, L13

Kochukhov, O. 2013, arXiv:1309.6313

Landstreet, J. D. 1982, ApJ, 258, 639

Lignières, F., Petit, P., Böhm, T., \& Aurière, M. 2009, A\&A, 500, L41

López Ariste, A. 2002, ApJ, 564, 379

Makaganiuk, V., Kochukhov, O., Piskunov, N., et al. 2011, A\&A, 525, A97

Michaud, G., Charland, Y., Vauclair, S., \& Vauclair, G. 1976, ApJ, 210, 447

Michaud, G. 1970, ApJ, 160, 641

Monnier, J. D., Che, X., Zhao, M., et al. 2012, ApJL, 761, L3

Petit, P., Lignières, F., Aurière, M., et al. 2011, A\&A, 532, L13

Petit, P., Lignières, F., Wade, G. A., et al. 2010, A\&A, 523, A41

Petit, P., Donati, J.-F., Aurière, M., et al. 2005, MNRAS, 361, 837

Royer, F., Zorec, J., \& Gómez, A. E. 2007, A\&A, 463, 671

Shorlin, S. L. S., Wade, G. A., Donati, J.-F., et al. 2002, A\&ऽA, 392, 637

Takeda, Y., Kawanomoto, S., \& Ohishi, N. 2008, ApJ, 678, 446

Tutukov, A. V. \& Fedorova, A. V. 2010, Astronomy Reports, 54, 156

Wade, G. A., Grunhut, J., Alecian, E., et al. 2013, arXiv:1310.3965

Wade, G. A., Aurière, M., Bagnulo, S., et al. 2006, A $\mathscr{E} A$, 451, 293 